areas of need identified, we worked with a training provider to develop a bespoke training course on supporting people with dementia in palliative care. The course was delivered to 41 staff within a hospice, and the impact assessed. Prior to training, 30\% of staff reported they were 'not at all confident' in caring for people with dementia. Post-training all staff reported feeling 'fairly' or 'very' confident. Staff reported that the training:

- Helped them recognise dementia as a terminal condition and how palliative care can support people with dementia

- Improved understanding of 'challenging' behaviours and managing these

- Taught them enabling approaches to care provision which they felt would improve their practice.

Staff trainers across both settings are integrating this into core curriculum. The positive response represents a culture change. Staff better understand the value of palliative care for people with dementia and are motivated to explore how care practices can be improved for people with dementia. The organisation recognises this work as best practice and are exploring its potential as a national training standard.

\section{STARTING A COLLABORATIVE RESEARCH IMPACT ASSESSMENT: A TALE OF TWO RESEARCH FUNDERS}

${ }^{1}$ Kay Lakin, ${ }^{2}$ Sanjay Thakrar, ${ }^{1}$ Claire Vaughan, ${ }^{2}$ Sabine Best, ${ }^{2}$ Laura Chatland, ${ }^{1}$ Adam Lockwood, ${ }^{1}$ Howard Simons, ${ }^{1}$ Sarah Thomas, ${ }^{1}$ Sam Watson, ${ }^{3}$ Sam Ahmedzai, ${ }^{3}$ Shamaila Anwar. ${ }^{1}$ National Institute for Health Research, UK; ${ }^{2}$ Marie Curie, UK; ${ }^{3}$ National Institute for Health Research Clinical Research Network

10.1136/spcare-2019-mariecuriepalliativecare.14

The National Institute for Health Research (NIHR) and Marie Curie are the two largest funders of end of life care research in the UK. NIHR and Marie Curie enable and support research at different times of the translational pathway, or by co-funding research. Undertaking a research impact assessment (RIA) on our combined investment is important to make the case for continued investment research and to be accountable to our stakeholders (Morgan Jones and Grant, 2013). RIA is a developing field (Adam et al., 2018). To ensure our approach is relevant, we have involved people with experience of end of life care. We hope that by undertaking this process together, we will gain a better understanding of the research funding landscape and its interdependencies in this area, identify gaps and opportunities, and learn from and support each other. We believe that our project marks the first time that a collaborative RIA of this kind has been undertaken by research funders. We hope that by exchanging, reflecting and sharing our experiences, lessons learned and approach, it will aid discussion, promote shared learning and transparency around funders' impact assessment processes and may help future collaborative projects. Our learning so far:

- Appropriate oversight, governance and data sharing is a must

- Identify organisational risks up front

- Review organisational factors including resource which may impact on capacity to deliver

- Use of similar tools for data collection enables joint analysis and efficient of data

- collection
- A clear and shared understanding of the project scope is important

\section{A 'GOOD' DEATH IN THE EMERGENCY DEPARTMENT? IDENTIFYING PATIENTS NEARING THE END OF LIFE IN THE ED}

Renate Fromson. The Whittington Hospital, Whittington Health NHS, London, UK

10.1136/spcare-2019-mariecuriepalliativecare.15

An important aspect of end of life (EOL) care is identifying patients entering this stage, thus enabling clinicians to make plans and referrals. Despite many patients wishing to die at home, a significant proportion will attend the ED. Hence it is important that ED doctors identify and support these individuals. Given the nature of work in ED, this is challenging. The Supportive and Palliative Care Indicators Tool (SPICT ${ }^{\mathrm{TM}}$ ) can help with patient identification.

A sample of 30 patients aged $\geq 60$ attending the Whittington Hospital ED were assessed using the SPICT ${ }^{\mathrm{TM}}$ criteria. For patients who met EOL criteria, it was documented what action was taken, including Advanced Care Planning, DNACPR, referral to Palliative Care or discussion about preferred place of care and of death.

10 met the criteria. Action was taken in $40 \%$ of these, most commonly Advanced Care Planning and DNACPR discussion. Use of the SPICT ${ }^{\mathrm{TM}}$, in poster or app format, could help ED doctors to identify EOL patients, thus prompting them to take action.

Further ways to improve the identification of EOL patients in the ED include use of tools such as the 3 triggers outlined by the GSF Prognostic Indicator Guide for GPs. Other ideas include an alert system to flag patients known to Palliative Care. Rapid discharge pathways for patients who wish to die at home is also an important initiative which relies on rapid identification and close working with community teams.

\section{$16 \quad$ IMPROVING CARE FOR PEOPLE LIVING AND DYING WITH DEMENTIA THROUGH ADVANCE CARE PLANNING}

Julie Skelton. Marie Curie Cardiff and The Vale Hospice, Cardiff, UK

\subsection{6/spcare-2019-mariecuriepalliativecare. 16}

The number of people living and dying with dementia is forecast to increase by $270 \%$ over the next 20 years. Current practices to encourage advance care planning do not meet the needs of people with a dementia diagnosis as they don't engage people early enough in the dementia journey.

We undertook a literature review of international evaluated models of ACP for people with dementia, focusing on its impact on care received, and mapped existing provision across a rural health board area. The work was facilitated by interviews with health and social care professionals $(n=50)$, focus groups and interviews with people living with dementia and carers $(n=17)$.

Our findings

- ACP is an intervention which can reduce unnecessary hospital admissions and healthcare costs, without negatively impacting 
quality of care and aligns to the principles of prudent healthcare.

- Recognition that dementia is a terminal illness and early engagement about ACP is fundamental to improving end of life care.

- Talking about ACP requires time, knowledge and sensitivity. Everyone who interacts with the person with dementia has a role to play in ensuring an ACP discussion takes place.

- A structured approach is required to prepare, equip and support health and social care professionals to progress the discussion and the equally important documentation and communication of wishes.

Good practice is an end-to-end process which is valued and understood by everyone who is part of it. It is reliable and robust; health and social care workers feel informed, supported, protected and empowered to meet the patient's wishes.

\section{PALLIATIVE RADIOTHERAPY}

1,2Linda Bedford. 'Macmillan Cancer Support, UK; ${ }^{2}$ Radiotherapy Dept., Beacon Centre, Musgrove Park Hospital, Taunton and Somerset NHS Foundation Trust, Taunton, Somerset, UK

\subsection{6/spcare-2019-mariecuriepalliativecare.17}

Introduction Radiotherapy treatment with palliative intent accounts for $35-40 \%$ of the radiotherapy department's workload. Historically, patients had a long wait to see Consultant Clinical Oncologists and receive radiotherapy for their symptoms (the most common of which is pain) (Jones et al 2014).

Service Improvement A service has been developed for rapid access to palliative radiotherapy, to improve the patient experience and access timely symptom control. This is achieved with radiographer led service where patients are seen by a Consultant Radiographer (CR), assessed for radiotherapy, consented, referred and radiation prescriptions all completed eliminating waiting times for Oncologists clinics. Including a CR led outreach service for the trust and local Hospices ensuring all patients requiring palliative radiotherapy receive timely assessment and treatment.

Aims

- Assess patients individually in terms of suitability for palliative radiotherapy.

- Promote awareness of Palliative Radiotherapy as a treatment option in managing

- metastatic cancer as a complex long term condition

- Reduction of Pill Burden (Farrell et al 2013)

- Reducing length of stay and admissions.

- Holistic pain management - liaising with palliative care teams in the community.

Results A streamlined process has been developed demonstrating continuity of care. Specialist nurses and physios now contact the CR for advice and direct referrals. Pain is managed in a timely manner.

Conclusion This work has highlighted the complex nature of palliative radiotherapy in the management of cancer as a long term condition enabling patients to access timely symptom control. There are still many challenges to overcome and this is very much work in progress.

\section{USING ROUTINE DATA TO IDENTIFY THE FACTORS THAT PLACE PATIENTS AT RISK OF NOT RECEIVING PALLIATIVE CARE}

${ }^{1}$ Peter Edmunds, ${ }^{2}$ Mirella Longo. 'Vancouver Coastal Health, Vancouver, Canada;

${ }^{2}$ University of Cardiff, Marie Curie Palliative Care Research Centre, UK

\subsection{6/spcare-2019-mariecuriepalliativecare.18}

Introduction Despite the benefits and growing acceptance that palliative care should be made available to seriously ill, frail and dying people (Kavalieratos et al. 2016, Singer et al. 2016), for some, this is still not the case. This study used historical routine data to determine the factors associatedwith not receiving palliative care.

Methods Four electronic healthcare databases (Discharge Abstract Database recording Hospital care, Emergency Department Database, PARIS community care database, Palliative Care patient registry database) were linked for all deceased patients over 2 years in a suburban population of 200,000 in Vancouver, Canada.

Results 2504 deceased individuals were identified. Of these, 2424 patients could be linked and assigned to a leading cause of death (761 cancer, 689 dementia/frailty, 974 organ failure). $67 \%$ of all patients had a record of receiving palliative care, however, almost half of these received less than 56 days of palliative care.

For people facing end of life illness, factors increasing the risk of not being offered palliative care were:

- No attachment to a general practitioner,

- Organ failure/non-malignant diagnoses,

- Males with organ failure/non-malignant diagnoses,

- Very low median household income and,

- Patients with dementia living at home.

Conclusion With the use of routine data it is possible to proactively identify and respond to patients in high-risk categories, reducing reliance on referral by clinicians. Routine data can be used to predict the palliative care needs of specific geographical areas.

\section{USING PROJECT ECHO TO MEET THE DEMAND FOR PALLIATIVE CARE EDUCATION AND SUPPORT ACROSS MULTIPLE SETTINGS}

${ }^{1}$ Janet Diffin, ${ }^{1}$ Tracey McTernaghan, ${ }^{2}$ Aine McMullan, ${ }^{2}$ Martin Hayes ${ }^{1}$ Max Watson. ${ }^{1}$ Hospice UK, UK; ${ }^{2}$ Health and Social Care Board UK

10.1136/spcare-2019-mariecuriepalliativecare.19

Background Despite increasing demand, limited resources are often available to deliver high quality palliative care education. Project ECHO is a tele-mentoring programme that could address this disparity by using video-conferencing to deliver (i) best practice guidance and education from specialists, and (ii) case-based learning with peer discussion1. Five ECHO networks in Northern Ireland delivered palliative care education and support (Cardiology/Heart Failure, District nurses, Community pharmacy, Paediatrics, Marie Curie registered nurses). Participants set the curriculum and network aims and objectives, which included increasing participant's palliative care knowledge and skills, enhancing confidence, improving collaboration, and facilitating peer support. 45 ECHO sessions were delivered across five networks with 194 participants attending $\geq 2$. 